\title{
Atenuación en textos informativos de prensa
}

\author{
ON LINGUISTIC MITIGATION IN NEWS ITEMS
}

\section{Paula Bouzas Rosende}

Georg-August-Universität Göttingen, Alemania

Resumen:

El presente artículo se enmarca en el estudio de la atenuación y se centra en el análisis de este fenómeno en los textos informativos de prensa. El análisis, de carácter cualitativo y efectuado sobre un corpus de textos informativos procedentes de tres diarios españoles, atiende a los factores clave en el proceso de atenuación (los recursos atenuantes, los elementos causantes y los elementos atenuados), así como a las diversas funciones de atenuación detectadas. El análisis se basa fundamentalmente en las premisas formuladas por Caffi (2007), Albelda y Briz (2013) y Albelda y otros (2014). El objetivo concreto del trabajo es ofrecer una descripción sistematizada de las operaciones atenuantes registradas.

PALABRAS ClaVe: atenuación, prensa, textos informativos, análisis cualitativo.
Abstract:

This article is in line with mitigation studies and focuses on the analysis of this phenomenon in news items. A qualitative analysis has been performed on a corpus of news items from three Spanish newspapers to unravel the key factors of the mitigation process (attenuating resources, triggers and mitigated elements). Likewise, the different attenuating functions indentified were also studied. The analysis is based primarily on the premises of Caffi (2007), Albelda y Briz (2013) and Albelda y otros (2014). The concrete goal of this study is to offer a systematized description of the mitigation strategies found in the corpus.

KEYWORDS: mitigation, press, news items, qualitative analysis. 


\section{INTRODUCCIÓN}

El centro de esta investigación es el estudio de la atenuación en los textos informativos de prensa, textos de carácter narrativo o descriptivo en los que, al contrario de lo que ocurre en los argumentativos o los dialógicos en ámbitos formales, no se espera una presencia importante de elementos atenuantes (Albelda y Briz, 2013: 298). El análisis de estos recursos en los textos periodísticos de carácter informativo, sin embargo, permitirá aportar datos interesantes al estudio general de la atenuación así como poner de manifiesto la existencia de estrategias y motivaciones en la transmisión de información que van más allá de las relacionadas con la mera descripción de hechos.

El estudio tiene carácter cualitativo y se basa en un minicorpus de 88 artículos extraídos del Corpus de Referencia del Español Actual (CREA). De esta fuente se han tomado los textos informativos (noticias y reportajes) publicados entre 1996 y 2012 en tres diarios de difusión suprarregional (El País, El Mundo, $A B C$ ) en torno a un tema específico: la representación de las lenguas que conviven en España con el español en un estatus de cooficialidad (Bouzas, 2016; Bouzas, en prensa).

El objetivo concreto de este trabajo es la identificación, descripción y sistematización de los factores clave en los procesos atenuantes presentes en el corpus analizado. En primer lugar, se llevará a cabo una descripción y análisis de los factores más relevantes en el proceso de atenuación, en concreto: los recursos atenuantes, los elementos desencadenantes y los elementos atenuados. A continuación, se especificarán las funciones que cumplen los procesos de atenuación observados. Como paso previo necesario a la exposición de los resultados del análisis, se aclararán algunas premisas teóricas y metodológicas. Por último, a modo de síntesis y conclusión, se retomarán los aspectos que han resultado más relevantes en la caracterización, así como el esquema clasificatorio propuesto para englobar el fenómeno.

\section{PREMISAS TEÓRICAS}

\subsection{Sobre el concepto de atenuación}

El fenómeno de la atenuación es abordado desde una perspectiva pragmático-retórica (Caffi, 2007: 93-94) como estrategia lingüística de distanciamiento del mensaje con el fin de persuadir y lograr un determinado objetivo en tanto que se cuidan expectativas sociales y relaciones personales (Albelda y Briz 2013: 292). En este sentido, la atenuación incide lingüísticamente de diferentes maneras (Briz, 2006; Albelda y Briz, 2013: 293; Caffi, 2013: 270): 1) mitigando el contenido del enunciado para evitar efectos no deseados, 2) mitigando la fuerza ilocutiva de los actos de habla y 3 ) reduciendo (o desfocalizando) el papel de los participantes en la comunicación.

Conforme a ello, Caffi (2007: 98-107) distingue y describe tres tipos de estrategias atenuantes: bushes, hedges y shields. Con el término bush son denominadas aquellas estrategias que inciden en el contenido de la proposición reduciendo el grado de precisión, mientras que el término hedge remite a las estrategias que inciden en el acto ilocutivo, 


\section{normas}

Atenuación en textos informativos de prensa | Paula Bouzas Rosende

mitigando su fuerza ${ }^{1}$. El término shield, por su parte, hace referencia a estrategias que afectan a la entidad enunciadora e implican una despersonalización de la misma o su desplazamiento a un segundo plano. Caffi menciona además dos estrategias de atenuación que ella denomina quotational shields y topical shields respectivamente (Caffi, 2007: 114-119). El quotational shield consiste en la introducción de elementos metalingüísticos que dejan en suspenso el significado literal del enunciado, lo que a su vez implica una suspensión del compromiso con el enunciado por parte de la entidad emisora. La estrategia de topical shield, por su parte, está orientada a reducir el grado de relevancia del tema para el propósito presente de la comunicación y se produce, según la autora, en forma de digresión o ejemplificación estratégica.

La propuesta de Caffi (2007) supone una ampliación de modelos anteriores de carácter bipartito. Uno de ellos es el de Prince y otros (1982: 85), en el que se distinguen dos tipos de mitigación (fuzziness): una tiene lugar en el plano del contenido proposicional y la otra incide en la relación entre este contenido y la entidad emisora, concretamente, en el compromiso de la entidad emisora con la veracidad de la proposición. Con respecto a este segundo tipo, para el que los autores reservan el término shield, se establece una importante distinción entre plausibility shields y attribution shields. Los primeros se refieren a las expresiones de duda, mientras que los segundos se corresponden con expresiones a través de las cuales una entidad emisora atribuye el contenido proposicional a otra persona. En la terminología de Caffi (2007), el primer tipo de recursos ilocutivos serían denominados hedges, mientras que los segundos mantendrían la denominación de shields.

En lo referente a los actos de habla asertivos, como los que se producen en la situación comunicativa analizada en este trabajo, Caffi señala el predominio de lo que ella denomina atenuación temperativa (Caffi, 2001: 253, 279; 2007: 83), que consiste fundamentalmente en la mitigación del compromiso epistémico de la entidad emisora con la verdad de la proposición o la reducción de la carga categórica del mensaje. Con ello, se reduce para la entidad receptora la obligación de creer y, al mismo tiempo, se elude por parte de la entidad emisora una amenaza potencial a la propia imagen. En conclusión, la atenuación temperativa afecta fundamentalmente a la relación entre la entidad emisora y el objeto de su enunciado e implica una atenuación en el plano del contenido proposicional (el plano semántico de la expresión) y de la ilocución (la intención comunicativa subyacente, en este caso siempre asertiva).

\subsection{Sobre los conceptos de enunciado y aserción}

Los enunciados presentes en los textos informativos de prensa son emitidos por una entidad periodística, a la que nos referiremos con el término entidad enunciadora o emisora, cuya intención, en primera instancia, es ofrecer información sobre determinados hechos de interés. Esta entidad enunciadora asume la responsabilidad sobre el enunciado emitido y, por lo tanto, sobre su veracidad ${ }^{2}$. Este aspecto determina que los enunciados

\footnotetext{
${ }^{1}$ Cfr. el uso del término en Lakoff (1972), Fraser (1975) y Brown y Levinson (1978).

${ }^{2}$ Aunque la polifonía es una característica del tipo de texto analizado, no se atenderá aquí a la distinción entre locutor vs. enunciador (cfr. Ducrot 1986; Fuentes Rodríguez 2004). En los numerosos casos de discurso reproducido que se tratarán en el análisis, la noticia transmitida por la entidad periodística no consiste en la información trasmitida por otra entidad
} 
en cuestión se adscriban a un acto de habla asertivo y, en concreto, asertivo de la información (Albelda y otros, 2014: 49-51). La aserción se entiende pues como el acto ilocutivo en el cual una entidad emisora informa a la entidad interlocutora de algo cuya verdad asume (Grande Alija, 2002: 216; Fuentes Rodríguez, 2004: 125-126).

Con el término enunciado se denomina el resultado o producto lingüístico derivado de la actividad comunicativa, en oposición a enunciación (Ducrot, 1986: 183; Fuentes Rodríguez, 2004). El enunciado es, conforme a Ferrari y otros (2008: 31), la realización locutiva de una unidad comunicativa que se corresponde a su vez con una determinada funcion ilocutiva y una determinada función textual. El núcleo, unidad informativa principal del enunciado, determina las funciones ilocutiva y textual de toda la unidad comunicativa y puede ir acompañado de unidades informativas facultativas (Ferrari y otros, 2008: 45-49): el marco (quadro), que define el dominio semántico-pragmático en el que se debe adscribir el núcleo, y el apéndice (appendice), unidad subordinada al marco o al núcleo y del mismo tipo que la unidad a la que se subordina. Esta distinción entre diferentes unidades informativas resultará útil a la hora de describir algunos de los procesos atenuantes observados.

\section{DESCRIPCIÓN DEL CORPUS Y CUESTIONES METODOLÓGICAS}

Para la realización del análisis se toma como referencia la ficha metodológica propuesta por Albelda y otros (2014). En ella se tienen en cuenta los siguientes factores de análisis: función de la atenuación, procedimientos lingüísticos atenuantes, elementos estructurales (elemento causante, segmento atenuante y segmento atenuado), factores enunciativos (imágenes, fuerza ilocutiva) y factores situacionales (temática, interacción, contexto físico, características de las personas participantes, etc.). El presente análisis se centrará en los tres primeros factores: procedimientos lingüísticos atenuantes, elementos estructurales y función de la atenuación.

En lo que respecta a los factores restantes mencionados en la ficha, de carácter enunciativo y situacional, el corpus se caracteriza por su homogeneidad. En cuanto al plano enunciativo, se trata de noticias de prensa orientadas, en principio, a la descripción de hechos, algunas veces narrativizados, en las que predomina, como hemos mencionado, el acto de habla asertivo de información. La entidad enunciadora, en muchos casos colectiva y anónima, es profesional del periodismo. Para que el público acepte su texto como veraz, así como para mantener su imagen, debe respetar las expectativas sociales inherentes a este intercambio, entre ellas, la apariencia de equidistancia o neutralidad. En cuanto al plano situacional, nos encontramos ante situaciones comunicativas en las que prima una finalidad transaccional (ofrecer información, lograr convencer), desarrolladas en un marco no-físico y protagonizadas por entidades interlocutoras entre las que existe una relación no-vivencial, no-bidireccional y asimétrica.

Con respecto a su contenido, se trata de textos de temática social o política (es decir, no especializada, no ritualizada, no cotidiana). En ellos se describen eventos de diferente naturaleza, en concreto, proposiciones de ley, resoluciones judiciales o avance de 
ponencias, pero también se incide en las actitudes o reacciones de colectivos o individuos, sobre todo desde el ámbito de la política, ante declaraciones de otras personas o ante determinadas cuestiones (política lingüística, financiación autonómica o medios de comunicación).

Precisamente la relevancia que cobran las manifestaciones de determinados agentes explica que una de las características fundamentales de los textos informativos de prensa sea la presencia de discurso reproducido. A la hora de aplicar la metodología y efectuar el análisis, es necesario distinguir en este sentido tres posibilidades: 1) enunciados en estilo directo, 2) enunciados en estilo indirecto, libre o narrativizado y 3) palabras o expresiones entrecomilladas tomadas, en principio, de otro discurso. Los enunciados del primer grupo, introducidos en estilo directo, no se han tenido en cuenta, puesto que no pertenecen a la entidad periodística enunciadora. Los enunciados del segundo grupo implican cierta dificultad metodológica, puesto que, en muchos casos, es imposible determinar si el recurso atenuante estaba presente en el enunciado original o no. Estos casos sí se incluyen en el análisis porque en cualquier caso son enunciados efectuados por la entidad emisora periodística. También son objeto de análisis las formas del tercer grupo, porque, aunque se trata de expresiones formuladas por otra entidad, en ocasiones son introducidas e incluso adaptadas en el enunciado propio. En este caso, además, el uso de comillas puede funcionar como recurso atenuante, en tanto que le permite a la entidad emisora introducir expresiones de terceras personas para elaborar su discurso al tiempo que salvaguarda su propia imagen de neutralidad.

En el siguiente apartado se detallarán los recursos atenuantes identificados, así como los factores estructurales más relevantes en el proceso de atenuación y las funciones de atenuación observadas.

\section{DESCRIPCIÓN DE LOS RESULTADOS DEL ANÁLISIS}

\subsection{Recursos atenuantes}

En primer lugar, se presentarán los recursos atenuantes registrados desde un punto de vista meramente formal. Tras la descripción de los elementos causantes de la atenuación y de los elementos atenuados, se ofrecerá una propuesta clasificatoria de carácter funcional, en la que se tendrán en cuenta los factores anteriores y las premisas teóricas expuestas. La intención es ofrecer una descripción lo más detallada posible y contribuir al estudio de los procesos de atenuación en su complejidad.

\subsubsection{Clasificación formal}

Se clasificarán los recursos atenuantes, conforme a sus características formales, en las siguientes categorías: recursos léxicos, recursos morfológicos, recursos sintácticos, recursos de construcción textual y, por último, recursos gráficos. 


\subsubsection{Recursos léxicos}

- Cuantificadores de minimización o aproximativos ${ }^{3}$

Debilitan la carga semántica negativa de la forma a la que acompañan situándola en el extremo mínimo de una escala de cantidad:

(1) Respecto a la posibilidad de que este nuevo enfrentamiento con los socios nacionalistas del Gobierno puede ayudar a minar un poco unas relaciones que no pasan por su mejor momento, indicaron [representantes del PP] que hay asuntos en los que el PP no debe ceder (ABC 12.11.1997).

(2) El Estado español sería el que tuviese que responder en caso de que el plan de la Generalidad de imponer el etiquetado de productos en catalán se convirtiese en un conflicto ante el Tribunal de Luxemburgo con unas posibilidades casi nulas de ser ganado (ABC 01.10.1997).

- Expresiones difusoras del significado

Relativizan el significado literal de la forma a la que acompañan, introduciendo vaguedad en la expresión. Este tipo de recurso permite a la entidad emisora introducir una forma léxica como últimatum (vid. 3), cuyo significado incluye el rasgo 'absoluto, definitivo, terminante', al tiempo que minimiza su responsabilidad por la dimensión categórica de la elección léxica:

(3) Tras el fracaso del primer plan de euskaldunización [...] se pone en marcha este Segundo Plan, una especie de ultimátum para todos salvo para los altos cargos y las excepciones, ya existentes en el primer plan, como los funcionarios mayores de cuarenta y cinco años (ABC 20.04.1997).

- Expresiones de objetivación

En tanto que el mensaje se presenta como algo evidente, compartido por los demás, el papel de la entidad enunciadora como entidad informativa se diluye. En (4) se introduce la fórmula objetivadora en el marco del enunciado, segmento que determina el ámbito semántico-pragmático del núcleo de la unidad comunicativa (Ferrari y otros, 2008: 45-46):

(4) Como se sabe, Pujol aboga por el reconocimiento de Cataluña con una fórmula similar a la del Quebec (EM 20.04.1996).

- Procedimientos de carácter sustitutivo

Se trata de procedimientos orientados a evitar la presencia de un lexema muy marcado por su carga semántica negativa. En el corpus se detectan de forma recurrente dos procedimientos de este tipo: el eufemismo y la lítotes.

El eufemismo implica la utilización de un lexema con carácter semántico neutral:

(5) En cuanto al calificativo de «gansteril» que le dedicó el portavoz del Gobierno valenciano, Esteban González, Carod Rovira contestó que «yo me sitúo en otro nivel intelectual y tan bajo no llego» (EM 10.11.2004).

\footnotetext{
${ }^{3}$ En los ejemplos, siguiendo las pautas recomendadas en Albelda y otros (2014), los elementos atenuantes se indican en negrita y, cuando es pertinente, se señalan los elementos atenuados con letra cursiva.
} 


\section{normas}

Atenuación en textos informativos de prensa | Paula Bouzas Rosende

(6) El presupuesto anual de TV5 Monde es de 80 millones de euros, una cifra modesta, pero que se explica porque parte de la programación no le cuesta nada a la cadena (EP 02.11.2005).

(7) La medida obedece a la reestructuración en la que está inmersa Radio Televisión Española, lo que incluye una disminución de la plantilla de 4.150 trabajadores (ABC, 08.01.2007).

La lítotes implica bien la negación de un lexema o expresión con significado positivo (vid. 8, 10: no ver con buenos ojos, no pasar por su mejor momento) o bien su degradación en la escala de cantidad mediante la introducción de modificadores (vid. 9, 11: escasas, poco $)^{4}$ :

(8) El director general de Euskal Irrati Telebista [...] no termina de ver con buenos ojos la posibilidad de privatizar (EM 10.05.1996).

(9) La proposición de ley del grupo parlamentario del PNV (...) tiene escasas posibilidades de prosperar (EP, 16.04.1997).

(10) Respecto a la posibilidad de que este nuevo enfrentamiento con los socios nacionalistas del Gobierno puede ayudar a minar un poco unas relaciones que no pasan por su mejor momento, indicaron [representantes del PP] que hay asuntos en los que el PP no debe ceder (ABC 12.11.1997).

(11) La ponencia del Estatuto de Cataluña dejó ayer cerrado el Título I, regulador de los «derechos y deberes» de los catalanes, tras una sesión poco pacífica (ABC 14.02.2006).

\subsubsection{Recursos morfológicos}

- Uso del tiempo verbal condicional

Es el único recurso de carácter morfológico detectado. Evidentemente, no todos los casos de uso de condicional implican atenuación. Se observa, sin embargo, la presencia de esta dimensión en aquellos casos en los que la forma carece de carácter deíctico y de motivación gramatical. Su función es la de debilitar el compromiso de la entidad emisora con la verdad del mensaje transmitido y, por lo tanto, reducir el carácter asertivo del enunciado:

(12) Los sondeos de opinión publicados en estas fechas no parecen acercarse a lo que la Coalición Popular consideraría unos resultados «de éxito» (ABC 16.06.1996).

(13) El Estado español sería el que tuviese que responder en caso de que el plan de la Generalidad de imponer el etiquetado de productos en catalán se convirtiese en un conflicto ante el Tribunal de Luxemburgo (ABC 01.10.1997).

(14) La referencia que hace el texto a esos aspectos, aunque sea matizada, justifica el rechazo que mantiene el PP al proyecto, aunque en última instancia podría optar por la abstención (ABC 09.11.1997).

En todos los ejemplos aducidos, habría sido posible la aplicación de un tiempo verbal con el rasgo [+ real]. En (12) el condicional sustituye a un tiempo presente. En (13) la

\footnotetext{
${ }^{4}$ Aunque el recurso de lítotes tiene efectivamente incidencia en el plano sintáctico, el hecho de que su funcionamiento implique la exclusión de determinadas formas léxicas y guarde paralelismos, como estrategia in absentia, con el eufemismo, explica su inclusión en esta categoría.
} 
existencia de una condición (en caso de que) hace redundante el uso del condicional y perfectamente válida la utilización de un tiempo con valor deíctico de futuro (será). En (14) la potencialidad de la acción viene expresada ya por una perífrasis modal poder + inf., así como por una expresión circunstancial en última instancia, por lo que hubiese sido posible optar por el uso del presente o del futuro de indicativo. El uso del condicional incide, por lo tanto, en primera instancia, en el compromiso de la entidad emisora con lo afirmado.

\subsubsection{Recursos sintácticos}

Se incluyen en este apartado los procedimientos de atenuación consistentes en la utilización de determinadas perífrasis o construcciones verbales, encabezadas por un verbo que asume una función auxiliar e introduce determinados matices.

- Construcciones verbales que expresan duda o probabilidad: poder + inf.

No siempre presenta este tipo de construcción un carácter atenuante, pero en algún caso es posible detectar un uso claramente estratégico. En (15) la dimensión atenuante de la perífrasis está determinada por la presencia de otros recursos atenuantes en el enunciado (el modificador un poco o la lítotes no pasar por el mejor momento) y, sobre todo, por la mención explícita a la probabilidad (respecto a la posibilidad) que le confiere a la perífrasis un carácter redundante:

(15) Respecto a la posibilidad de que este nuevo enfrentamiento con los socios nacionalistas del Gobierno puede ayudar a minar un poco unas relaciones que no pasan por su mejor momento, indicaron [representantes del PP] que hay asuntos en los que el PP no debe ceder (ABC 12.11.1997).

- Construcción verbal con un auxiliar evidencial

Se trata en este caso de la introducción de una expresión evidencial (Cornillie, 2016) que implica un modo de acceso inferencial, es decir, basado en una deducción o evaluación previa por parte de la entidad enunciadora. Con ello, se evita presentar como un hecho una determinada situación, claramente negativa para un determinado colectivo.

(16) Los sondeos de opinión publicados en estas fechas no parecen acercarse a lo que la Coalición Popular consideraría unos resultados «de éxito» (ABC 16.06.1996).

- Construcciones verbales con auxiliar de acción inacabada

Nos encontramos ante un caso concreto de circunloquio, parcialmente sustitutivo: el verbo que recoge la carga semántica referencial no aparece de forma autónoma y finita, sino formando parte de una construcción perifrástica (ayudar a + inf., no terminar de + inf.) cuyo auxiliar introduce un matiz aspectual:

(17) El director general de Euskal Irrati Telebista (EITB), Iñaki Zarraoa, no termina de ver con buenos ojos la posibilidad de privatizar (EM 10.05.1996).

(18) Respecto a la posibilidad de que este nuevo enfrentamiento con los socios nacionalistas del Gobierno puede ayudar a minar un poco unas relaciones que no pasan por su mejor momento, indicaron [representantes del PP] que hay asuntos en los que el PP no debe ceder (ABC 12.11.1997). 


\subsubsection{Recursos de construcción textual}

- Expresiones que acotan la opinión a terceras personas

En tanto que una determinada acción se presenta como una opinión atribuible a un grupo de personas, ajenas a la entidad enunciadora, esta evita comprometerse con su veracidad.

(19) Los sondeos de opinión publicados en estas fechas no parecen acercarse a lo que la Coalición Popular consideraría unos resultados «de éxito» (ABC 16.06.1996).

- Introducción de construcciones subordinadas

Se trata en este caso de introducir determinadas cláusulas que matizan o restringen el carácter potencialmente negativo de las acciones u opiniones descritas en el núcleo de la unidad comunicativa. Las unidades sintácticas que pueden cumplir esta función en el corpus son:

- Construcciones adversativas

(20) El presupuesto anual de TV5 Monde es de 80 millones de euros, una cifra modesta, pero que se explica porque parte de la programación no le cuesta nada a la cadena (EP 02.11.2005).

- Construcciones causales a modo de justificación

(21) Este informe que propone la mayoría conservadora [del CGPJ] rechaza que el conocimiento del catalán sea considerado como "capacidad", es decir, obligatorio, para los jueces que quieran ejercer en Cataluña, pues ello podría ser contrario a la Constitución (ABC 17.11.2004).

- Cláusulas subordinadas temporales

En el ejemplo aducido (22), mediante una cláusula temporal colocada entre comas a modo de paréntesis (vid. infra §2.2.1.5.), se redunda en el carácter cautelar de la decisión. Estos dos aspectos, el carácter redundante y su posición en el enunciado, apuntan a un valor atenuante que predomina frente al informativo:

(22) EI TSJC ha dictado un auto en el que acepta imponer como medida cautelar, mientras se resuelve un recurso judicial, la obligación de una escuela de Badalona a impartir clases en castellano (EM 02.02.2006).

- Estrategias de lateralización

Se trata de un recurso de carácter textual que llamaremos de lateralización y que consiste en transmitir información novedosa y relevante para la situación comunicativa en un nivel jerárquico secundario, no nuclear, de la estructura informativa, en concreto, como marco (Ferrari y otros, 2008: 46):

(23) Respecto a la posibilidad de que este nuevo enfrentamiento con los socios nacionalistas del Gobierno puede ayudar a minar un poco unas relaciones que no pasan por su mejor momento, indicaron [representantes del PP] que hay asuntos en los que el PP no debe ceder (ABC 12.11.1997). 


\section{normas}

Atenuación en textos informativos de prensa | Paula Bouzas Rosende

(24) En cuanto al calificativo de «gansteril» que le dedicó el portavoz del Gobierno valenciano, Esteban González, Carod Rovira contestó que «yo me sitúo en otro nivel intelectual y tan bajo no llego» (EM 10.11.2004).

Tanto en (23) como en (24), el hecho de que la información sea presentada por medio de cláusulas introducidas por una locución preposicional (respecto a, en cuanto a) contribuye a que sea percibida por la entidad receptora como algo conocido y, por lo tanto, a que pierda su carácter informativo.

\subsubsection{Recursos gráficos}

- Uso de comillas

La introducción de comillas en los textos analizados puede responder a dos tipos de función diferentes: 1) la suspensión del sentido literal de una determinada expresión o 2) la indicación gráfica del discurso directo, es decir, la indicación de que una forma o expresión está tomada literalmente del enunciado formulado por otra persona.

En la primera de las funciones, menos significativa numéricamente en el corpus, el uso de comillas es claramente un recurso atenuante que favorece la anulación del contenido potencialmente negativo de una expresión o enunciado ${ }^{5}$ :

(25) Los sondeos de opinión publicados en estas fechas no parecen acercarse a lo que la Coalición Popular consideraría unos resultados "de éxito» (ABC 16.06.1996).

(26) Desde que en 1991 conquistara la presidencia del PP en Cataluña [...], Aleix Vidal-Quadras se ha convertido en la «bestia negra» de los nacionalistas catalanes (EM 08.08.1996).

En la gran mayoría de los casos, sin embargo, el uso de las comillas se corresponde con la segunda de las funciones descritas: marcar el discurso directo. Si bien los enunciados tomados directamente de otras entidades enunciadoras, y ajenas por tanto al discurso periodístico, no son objeto del análisis, sí ha resultado relevante el estudio de aquellas situaciones en las que la expresión introducida entre comillas se limita a una o varias palabras y está integrada sintáctica e incluso morfológicamente en el enunciado último. En la mayoría de estos casos, la entidad enunciadora utiliza las comillas para introducir, con fines estratégicos, una expresión procedente de otro discurso y portadora de una carga semántica claramente negativa. Se trata de un recurso atenuante particular, consistente no en anular el contenido negativo de un lexema, sino en anular su adscrición a la entidad enunciadora del enunciado presente:

(27) Serra y el portavoz del grupo socialista en el Parlamento catalán, Joaquín Nadal, instaron a la coalición nacionalista para que arregle «el desbarajuste» creado (ABC, 16.06. 1986).

(28) De las propuestas presentadas en el Gobierno de Aragón sobre la situación del catalán, criticó algunas iniciativas "exóticas», como que sean los municipios los que decidan en la Franja acerca de la regulación del catalán (ABC 27.10.2005).

(29) Pero el mérito que CiU se atribuyó [...] fue minusvalorado por formaciones como IC-V, ERC o el propio PP, que no perciben en los añadidos de CiU sino «ambigüedades» que lejos de suprimir parte de lo regulado en el proyecto,

\footnotetext{
${ }^{5}$ Caffi (2007: 114-115) denomina literalmente con el término quotational shield a un conjunto de recursos lingüísticos que se orientan en este mismo sentido.
} 


\section{normas}

Atenuación en textos informativos de prensa | Paula Bouzas Rosende

añaden confusión con el único fin de tratar de "salvar la cara» (ABC 14.02.2006).

En estos casos, la estrategia sería propia de un tipo específico de atenuación en el plano de la ilocución, equivalente a la categoría denominada attribution shield en Prince y otros (1982: 85).

- Uso de comas

La introducción de determinados elementos entre comas, lo que en definitiva remite a un determinado modelo prosódico (pausa, carácter parentético), contribuye a dotar a estos elementos de un carácter explicativo. En (30) la expresión situada entre comas a modo de aclaración (apéndice) introduce una reserva, esto es, una restricción al contenido transmitido por la cláusula principal (núcleo informativo):

(30) EI TSJC ha dictado un auto en el que acepta imponer como medida cautelar, mientras se resuelve un recurso judicial, la obligación de una escuela de Badalona a impartir clases en castellano (EM 02.02.2006).

\subsubsection{Clasificación en función del ámbito de incidencia}

Al comienzo de esta contribución, se han apuntado los tres grandes ámbitos o planos de incidencia de los procesos atenuantes: el contenido proposicional, la fuerza ilocutiva y el origen deíctico del enunciado. El intento de clasificar conforme a estas categorías los recursos atenuantes detectados ha planteado, no obstante, algunos problemas, por ejemplo, en lo que respecta al ámbito del origen deíctico. Recordemos que en este grupo estarían incluidos aquellos procedimientos de ocultación o difuminación que afectan a la entidad enunciadora y que funcionan estratégicamente, por lo tanto, como shields (Caffi, 2001; 2007). En nuestro corpus, además de un uso estratégico de las comillas para incluir en el enunciado propio expresiones concretas tomadas de enunciados ajenos (vid. 27-29), se registran dos recursos que inciden en este sentido:

- Expresiones que acotan la opinión a terceras personas

(31) Los sondeos de opinión publicados en estas fechas no parecen acercarse a lo que la Coalición Popular consideraría unos resultados «de éxito» (ABC 16.06.1996).

- Expresiones de objetivación

(32) Como se sabe, Pujol aboga por el reconocimiento de Cataluña con una fórmula similar a la del Quebec (EM 20.04.1996).

En ambos ejemplos, la entidad enunciadora se oculta para distanciarse del contenido del enunciado, pero el funcionamiento de la atenuación es muy diferente en cada uno de ellos. En el primero, en tanto que la entidad emisora lo presenta como una percepción ajena, se mitiga el carácter factual del contenido proposicional; en el segundo, al presentarse el contenido como un hecho obvio y compartido, se mitiga la relevancia informativa del mensaje (Albelda y otros, 2014: 35). Parece que estos ejemplos ponen de manifiesto la existencia de dos ámbitos diferentes de incidencia: la ilocución (en el primer caso) y la relevancia de la información (en el segundo). En hecho de que ambos recursos se orienten a la difuminación de la instancia enunciadora sería, conforme a esta 
interpretación y de cara a la clasificación funcional de los respectivos procedimientos atenuantes, de carácter secundario.

Al mismo tiempo, el ejemplo anterior muestra un tipo de atenuación consistente en reducir la relevancia informativa que tiene para el objeto de la comunicación una determinada unidad del enunciado (Caffi, 2007: 115). En aquel caso se utilizaba una expresión objetivadora (como se sabe) que incidía directamente, difuminándolo, en el papel de la entidad enunciadora. Pero en el corpus se registra, además, un recurso específico que propicia, de manera recurrente, la pérdida de relevancia informativa: la lateralización. Este procedimiento consiste en transmitir información novedosa y relevante para la situación comunicativa en un nivel jerárquico secundario de la estructura informativa:

(33) Respecto a la posibilidad de que este nuevo enfrentamiento con los socios nacionalistas del Gobierno puede ayudar a minar un poco unas relaciones que no pasan por su mejor momento, indicaron [representantes del PP] que hay asuntos en los que el PP no debe ceder (ABC 12.11.1997).

(34) En cuanto al calificativo de "gansteril» que le dedicó el portavoz del Gobierno valenciano, Esteban González, Carod Rovira contestó que «yo me sitúo en otro nivel intelectual y tan bajo no llego" (EM 10.11.2004).

La diferencia con respecto a otros procedimientos identificados como hedges o schields es que, en el caso de la lateralización, no hay una reducción del compromiso por parte de la entidad emisora con la veracidad de la aserción ni tampoco una difuminación de su papel como informante.

Teniendo en cuenta la descripción realizada de los factores más relevantes de atenuación en el corpus analizado, se efectuará a continuación una clasificación de los mismos en función de cuatro ámbitos de incidencia: 1) contenido proposicional, 2) fuerza ilocutiva, 3) entidad enunciadora y 4) relevancia informativa.

\subsubsection{El ámbito del contenido proposicional}

Los procedimientos incluidos en esta categoría actúan en el plano del contenido proposicional para mitigar rasgos semánticos negativos. En este sentido, distinguimos procedimientos aditivos y procedimientos sustitutivos: los primeros reducen la carga semántica de elementos presentes en el enunciado, mientras que los segundos consisten en evitar, por suplantación, la aparición de otras posibilidades léxicas con una carga semántica negativa más evidente.

Entre los procedimientos aditivos se pueden distinguir, adaptando la terminología de Blum-Kulka y otros (1989), recursos de atenuación interna (presentes en la propia unidad informativa sobre la que actúan) y recursos de atenuación externa (que actúan desde otra unidad informativa del mismo enunciado). Entre los primeros se encuentran modificadores y expresiones difusoras del significado que funcionan como bushes al introducir el rasgo semántico de vaguedad, así como también construcciones verbales con un auxiliar de acción incompleta (ayudar a + inf., no terminar de + inf.) que introduce en cuanto a la acción ese mismo matiz semántico. Como recursos de atenuación externa, se registran algunos procedimientos de construcción textual consistentes en la introducción de determinadas cláusulas (temporales, adversativas o concesivas) a modo de justificación, explicación o restricción del contenido transmitido por la cláusula principal.

En cuanto a los procedimientos sustitutivos que inciden en el contenido proposicional, se registran el eufemismo y la lítotes. Además, en relación con los recursos sustitutivos, cabe hacer mención a un caso especial: la elección de los verba dicendi a la 
hora de reproducir actos de habla ajenos que implican agresión. En nuestro corpus, concretamente, el uso de los verbos indicar y mencionar para describir actos de habla en los se manifiestan rasgos como [+ reproche] o [+ advertencia] responde a la intención de suavizar la fuerza ilocutiva con la que fueron emitidos los enunciados correspondientes, atenuar el carácter negativo del acto y preservar la imagen de la persona emisora original, como se puede observar en (35), (36) y (37). Se trata, pues, de una forma de sustitución léxica que, por su carácter metalingüístico, repercute en la percepción de la fuerza ilocutiva de mensajes formulados por otro locutor y enunciados nuevamente.

(35) Alberto Fernández le replicó ayer mismo indicando que algunos dirigentes de CIU añoran la etapa anterior a las últimas elecciones «cuando junto con el PSOE atacaban al Partido Popular» (EP 10.11.1997).

(36) La postura del Partido Popular respecto a esta ley [nueva ley del catalán] no ha cambiado [...] e indicaron que hay asuntos en los que el PP no debe ceder (ABC 12.11.1997).

(37) [Desde el Partido Popular] comentaron que mantendrán su «posición de firmeza» (ABC 12.11.1997).

En el contenido proposicional incide además una de las funciones observadas en el uso del recurso gráfico de las comillas (vid. 25, 26): al ser utilizadas para suspender el significado literal de una expresión, las comillas permiten atenuar o relativizar el valor semántico del lexema al que acompañan.

\subsubsection{El ámbito de la ilocución}

Los recursos se orientan en este caso a reducir la fuerza ilocutiva de la aserción informativa. En este sentido se pueden distinguir dos tipos de procedimientos:

a. Procedimientos orientados a reducir el rasgo [+ real] de la acción descrita.

Se trata de una serie de estrategias relacionadas con la introducción del rasgo modal de probabilidad o con la expresión de evidencialidad inferencial. Los recursos correspondientes son parcialmente sustitutivos, consistentes bien en la utilización de un determinado tiempo verbal (condicional) o en la utilización de una estructura analítica, con un verbo que actúa como auxiliar, en sustitución de otras alternativas posibles expresadas de forma sintética. En concreto, se trata de los siguientes recursos: el uso del tiempo verbal condicional (vid. 12-14), la perífrasis modal poder + inf. (vid. 15) y la construcción verbal con auxiliar evidencial parecer + inf. (vid. 16).

b. Procedimientos orientados a reducir el compromiso de la entidad enunciadora.

En este caso se trata de fórmulas que atribuyen a la opinión de terceros una determinada información (vid.19).

Esta distinción se equipara a las categorías que Prince y otros (1982) establecían al diferenciar entre plausibility shields y attribution shields, pero ambos tipos de recurso conllevan, en definitiva, el debilitamiento del carácter factual del enunciado.

\subsubsection{3 Ámbito de la entidad enunciadora}

El único recurso que incide funcionalmente en la difuminación de la entidad enunciadora a la hora de transmitir de forma asertiva un determinado contenido 


\section{normas}

Atenuación en textos informativos de prensa | Paula Bouzas Rosende

proposicional es el uso de comillas. Consideramos que este recurso implica un procedimiento estratégico de atenuación, puesto que afecta a lexemas concretos, muy marcados desde el punto de vista semántico (por su carga negativa o valorativa) o estilístico (vid. 27-29). La introducción de comillas en estos casos permite difuminar la figura de la entidad enunciadora, al tiempo que favorece la integración en el enunciado de lexemas que de otra manera no habrían sido aceptados en ese contexto comunicativo.

La diferencia con el tipo de atenuación ilocutiva (vid. §2.2.2.2) es que, en estos casos, no hay una mitigación de la factualidad del enunciado.

\subsubsection{4 Ámbito de la relevancia informativa}

Como se menciona más arriba, se trata en este caso de reducir la relevancia informativa de una determinada unidad del enunciado (Caffi, 2007: 115). Además de las estrategias de lateralización, habría que incluir aquí el uso de fórmulas objetivadoras (vid. 32), cuyo fin estratégico no es difuminar la figura de la entidad enunciadora, sino presentar el contenido proposicional como algo obvio y por lo tanto anular el carácter informativo de la aserción.

\subsubsection{Elementos causantes y elementos atenuados}

Para entender mejor cuál es el factor desencadenante de la atenuación en nuestro corpus y por qué, es importante retomar una consideración mencionada al comienzo de este trabajo: el tipo de texto analizado consiste en la descripción de eventos por parte de una entidad periodística y se caracteriza por estar dirigido a un público amplio cuya expectativa es recibir información. Aunque la entidad enunciadora es, en muchos casos, anónima, está sujeta al cumplimiento de estas expectativas y su imagen se sustenta, por lo tanto, en la informatividad de su texto y en su equidistancia. En otras palabras: se sustenta en su capacidad para presentar datos y hechos y hacer descripciones o hipótesis basadas en ellos. En este contexto, ciertos elementos semánticamente negativos determinan el enunciado emitido por la entidad enunciadora, en tanto que esta tratará bien de evitar en la medida de lo posible una descripción negativa de una situación o bien de mantener apariencia de objetividad.

Estos elementos pueden ser unidades léxicas o rasgos semánticos presentes en determinadas unidades o segmentos informativos. De acuerdo con esto, es posible establecer las siguientes categorías:

- Lexemas explícitos (in praesentia) con carga semántica negativa: ambigüedad, bestia negra, bloqueo, delirante, desbarajuste, desoladora, elevación de tensiones, empeorar, fracaso, fruto de violencia, minar, nulas, rechazo, ultimátum, etc.

Lexemas in absentia. Se trata en este caso de atenuar determinados rasgos semánticos negativos [+ conflicto], [+ rechazo] y [+ deterioro] evitando utilizar los lexemas correspondientes. En este grupo se incluyen asimismo rasgos semánticos inherentes a determinados verba dicendi ([+ reproche], [+ advertencia]) que son evitados mediante el uso de lexemas más neutros semánticamente, como indicar o comentar (vid. 35, 36, 37).

- Segmentos o unidades informativas que describen situaciones, acciones o actitudes ('ser algo de una determinada manera', 'tomar alguien una determinada decisión', 'tener alguien una cierta actitud ante algo o alguien'). Se caracterizan por la presencia de determinados rasgos semánticos negativos, más o menos explícitos, en el enunciado (vid. 20, 21, 22). 
En lo que se refiere a las formas explícitas, la atenuación se produce en dos tercios de los casos registrados mediante el uso de comillas. En los restantes casos, estas formas se atenúan en el plano del contenido proposicional mediante los siguientes recursos lingüísticos: 1) modificadores aproximativos y cuantificadores (minar un poco, posibilidades casi nulas, etc.), 2) expresiones difusoras del significado (una especie de ultimátum) y 3) auxiliares que expresan acción incompleta (ayudar a minar, (no) terminar de ver). Por su parte, sobre los elementos in absentia inciden los recursos sustitutivos del eufemismo (vid. 5-7) y la lítotes (vid. 8-11).

Por último, a la hora de atenuar unidades informativas, son aplicados recursos en los diferentes ámbitos de atenuación. En el plano del contenido proposicional, se produce atenuación al incorporar cláusulas adversativas, causales o temporales que restringen o matizan el contenido de la unidad informativa atenuada. En el plano de la ilocución (vid. $\S 2.2 .2 .2)$ se debilita el compromiso de la entidad enunciadora o la factualidad del contenido proposicional a través de recursos que afectan a la modalidad (uso del tiempo verbal condicional, introducción de la perífrasis modal poder + inf.), expresiones que indican evidencialidad inferencial (parecer + inf.) o referencias que acotadoras la opinión a terceras personas. Además, se reduce la relevancia informativa de estas unidades en tanto que se emplean fórmulas objetivadoras o estrategias de lateralización (vid. §2.2.2.3).

Aunque los procedimientos lingüísticos atenuantes indiden en varios ámbitos, el elemento causante de la atenuación en el corpus analizado es un elemento, más o menos explícito, presente en el enunciado y no tanto un rasgo abstracto del acto de habla (Caffi, 2013: 257). Ahora bien, la atenuación puede responder en cada caso a objetivos diferentes, por lo que es necesario analizar con detenimiento qué función cumple la atenuación en cada uno de los casos analizados. En otras palabras, es necesario, además del qué y del por qué, averiguar el para qué.

\subsection{Funciones de la atenuación}

Teniendo en cuenta la clasificación ofrecida por Albelda y otros (2014: 17-22), distinguimos en nuestro corpus las siguientes funciones:

\subsubsection{Protección / prevención sin imagen}

Aunque la imagen siempre está presente en la comunicación, hay casos en los que ciertamente se trata de controlar el alcance potencialmente negativo del contenido proposicional aun cuando este no supone una amenaza directa a la imagen de la entidad emisora, de la entidad receptora o de terceras personas. El objetivo último a la hora de aplicar la estrategia de atenuación en estos casos parece ser el de evitar que haya en la información determinadas implicaciones no deseadas que afectarían el modo en el que se percibe un estado de cosas.

Los recursos utilizados en este tipo de unidades comunicativas inciden fundamentalmente en el plano del contenido proposicional, bien mediante recursos sustitutivos como el eufemismo y la lítotes o bien mediante cláusulas temporales, causales o adversativas, que restringen el alcance semántico de la aserción principal:

(38) El presupuesto anual de TV5 Monde es de 80 millones de euros, una cifra modesta, pero que se explica porque parte de la programación no le cuesta nada a la cadena (EP 02.11.2005). 


\section{normas}

Atenuación en textos informativos de prensa | Paula Bouzas Rosende

(39) El director de los centros territoriales de RTVE, Manuel Lombao, explicó que la medida obedece a la reestructuración en la que está inmersa Radio Televisión Española, lo que incluye una disminución de la plantilla de 4.150 trabajadores (ABC 01.08.2007).

(40) El TSJC ha dictado un auto en el que acepta imponer como medida cautelar, mientras se resuelve un recurso judicial, la obligación de una escuela de Badalona a impartir clases en castellano (EM 02.02.2006).

\subsubsection{Protección de la propia imagen}

Se produce una protección de la propia imagen, salvaguarda del yo o curación en salud, relacionada con las convenciones sociales que deben ser respetadas por la entidad enunciadora en este contexto, a saber, neutralidad y equidistancia. Se suaviza el mensaje cuando el elemento con carga semántica negativa es explícito y, en última instancia, para facilitar su aceptación. En este sentido, es posible distinguir tres subfunciones específicas:

a. Evitar responsabilidades sobre lo dicho. Se trata de atenuar unidades léxicas explícitas, fundamentalmente mediante modificación externa o el uso de comillas:

(41) Serra y el portavoz del grupo socialista en el Parlamento catalán, Joaquín Nadal, instaron a la coalición nacionalista para que arregle «el desbarajuste» creado (ABC 16.06. 1986).

(42) Tras el fracaso del primer plan de euskaldunización [...] se pone en marcha este Segundo Plan, una especie de ultimátum para todos salvo para los altos cargos y las excepciones (ABC 20.04.1997).

b. Ser políticamente correcto. Recursos recurrentes en este tipo de atenuación son la lítotes y el eufemismo, es decir, los recursos sustitutivos en el plano del contenido proposicional:

(43) La ponencia del Estatuto de Cataluña dejó ayer cerrado el Título I, regulador de los «derechos y deberes» de los catalanes, tras una sesión poco pacífica (ABC 14.02.2006).

c. Evitar o minimizar responsabilidades en la emisión de pronósticos. En estos casos, sobre todo si el pronóstico es negativo, se echa mano de recursos que inciden en el plano ilocutivo y que contribuyen a reducir el rasgo [+ real] de la aserción:

(44) El Estado español sería el que tuviese que responder en caso de que el plan de la Generalidad de imponer el etiquetado de productos en catalán se convirtiese en un conflicto ante el Tribunal de Luxemburgo con unas posibilidades casi nulas de ser ganado (ABC 01.10.1997).

En el ejemplo aducido (44) se introducen además ciertos elementos que denotan vaguedad. Así, en el segmento final del enunciado, a la hora de informar sobre el hecho de que existen pocas posibilidades de ganar en el conflicto, la entidad enunciadora lleva a cabo primeramente un ejercicio intensificador, en tanto que utiliza un adjetivo con valor semántico de negación absoluta (nulas), que luego atenúa mediante la introducción de un modificador aproximativo (casi). 


\section{normas}

Atenuación en textos informativos de prensa | Paula Bouzas Rosende

\subsubsection{Prevención del daño a la imagen}

Se trata de evitar una amenaza para la imagen de otras personas y de prevenir un posible rechazo o el desacuerdo. En este sentido distinguimos dos subfunciones:

Evitar el rechazo o desacuerdo. La entidad enunciadora evita ir demasiado lejos en su descripción de una situación que podría ser negativa para ciertas personas. En última instancia, intenta evitar que el mensaje sea rechazado o que se origine un desacuerdo. Esta subfunción puede observarse en el siguiente ejemplo:

(45) Respecto a la posibilidad de que este nuevo enfrentamiento con los socios nacionalistas del Gobierno puede ayudar a minar un poco unas relaciones que no pasan por su mejor momento, indicaron [representantes del PP] que hay asuntos en los que el PP no debe ceder (ABC 12.11.1997).

En (45) se producen diversos recusos atenuantes que minimizan la carga semántica negativa presente en el verbo minar. Por una parte, se introduce el modificador un poco; por la otra, se reduce el rasgo [+ real] de la acción mediante la introducción de la perífrasis modal (poder + inf.). La utilización de un segundo verbo con función auxiliar (ayudar a + inf.) añade un matiz aspectual de acción inacabada y el matiz de vaguedad en la descripción de la acción. Además, en el enunciado está presente el recurso de la lítotes, mediante el cual se evita la utilización de un adjetivo de carga semántica negativa para definir la relaciones en cuestión ${ }^{6}$. Por último, hay que mencionar la lateralización de esta información en el enunciado: aun tratándose de contenidos nuevos y relevantes para el funcionamiento de la unidad comunicativa, estos son introducidos como marco (Ferrari y otros, 2008: 46) mediante la locución preposicional respecto a, como si se tratase de algo ya mencionado o conocido, de manera que su relevancia informativa queda mermada.

Prevenir dañar la imagen de otras personas. Esta función se detecta cuando, a través de la elección de determinados verba dicendi semánticamente neutrales (como indicar o comentar), se evita definir de forma negativa un acto de habla ajeno que incluye reproche o advertencia (vid. 35, 36, 37). De esta manera, se evita atribuir a los agentes una acción que podría dañar su imagen.

\section{SÍNTESIS Y CONCLUSIONES}

Se ha llevado a cabo el análisis cualitativo de los procesos atenuantes presentes en un minicorpus de 88 textos informativos de prensa. El objetivo era ofrecer una descripción y clasificación de los factores más relevantes en los procesos atenuantes (recursos atenuadores, elementos desencadenantes de la atenuación y elementos atenuados) presentes en los textos estudiados, así como de sus diferentes funciones.

En primer lugar, han sido presentados los recursos de atenuación, tanto gráficos como lingüísticos, desde un punto de vista formal. A continuación, se ha propuesto una clasificación basada en los ámbitos de indicencia, siguiendo modelos como los de Prince

\footnotetext{
${ }^{6}$ El uso estratégico de la lítotes en este caso, sin embargo, es ambiguo, puesto que es posible interpretar la existencia de una dimensión irónica, motivada por la aparición en el mismo enunciado de varios elementos léxicos que hacen explícita la carga semántica negativa del mensaje (nuevo enfrentamiento, minar).
} 
y otros (1982) y Caffi $(2001 ; 2007)$. En este sentido, se reconocen cuatro categorías principales: 1) procedimientos que actúan en el ámbito del contenido proposicional, 2) procedimientos que inciden en el ámbito de la fuerza ilocutiva, 3) procedimientos que repercuten en la relevancia informativa y 4) procedimientos que inciden en la entidad enunciadora.

Si bien no se trataba de ofrecer un análisis cuantitativo, es destacable el predominio de los recursos que inciden en el contenido proposicional, que se caracterizan tanto por su variedad como por su frecuencia. Entre ellos, se cuentan recursos aditivos, como modificadores, expresiones difusoras del significado o cláusulas que restringen el alcance semántico de la cláusula principal, y recursos sustitutivos (eufemismos y lítotes). Además, en el contenido proposicional incide el uso de las comillas cuando su función es la de suspender el significado literal de la expresión y relativizar su valor semántico (vid. 25, 26).

Menos frecuentes y variados son los recursos que actúan en el nivel ilocutivo. Al tratarse de textos informativos de prensa, entendemos que la reducción de la fuerza ilocutiva se puede producir en dos sentidos: 1) la reducción del rasgo [+ real] de la acción o 2) la reducción del compromiso de la entidad enunciadora con el contenido del enunciado. Ambos tipos de atenuación están orientados, en última instancia, a la mitigación del carácter factual del enunciado. Los recursos característicos de este tipo de atenuación son el uso del condicional, así como las construcciones perifrásticas con verbo auxiliar de carácter modal (poder) o evidencial (parecer). La reducción de compromiso se consigue mediante el uso de fórmulas acotadoras de la opinión para atribuir una información a terceras personas. Esta estrategia, que en principio podría estar orientada a ofrecer una crítica velada o a curarse en salud, contribuye, en combinación con otras estrategias (lítotes, reducción de la fuerza asertiva de la ilocución), a controlar el alcance negativo de una información en tanto que relativiza el carácter factual del contenido proposicional.

En lo que respecta a la difuminación del papel de la instancia enunciadora, el único recurso utilizado con fines estratégicos es el uso de comillas para integrar en el enunciado propio formas tomadas de otros enunciados. Su utilización para incorporar expresiones fuertemente connotadas semántica o estilísticamente es recurrente en el corpus.

Aunque se han identificado otros recursos que inciden sobre la entidad enunciadora, estos se orientan bien a la atenuación de su compromiso con el contenido de la proposición (como las fórmulas acotadoras de opinión a terceras personas), bien al debilitamiento de la relevancia informativa de su afirmación (como la expresión objetivadora como se sabe). En cuanto a este último ámbito de incidencia, la relevancia de un enunciado en la estructura informativa, se detectan en varias ocasiones estrategias de lateralización: ciertas informaciones sensibles en algún sentido son introducidas al comienzo de la estructura informativa en forma de cláusula subordinada por medio de una locución prepositiva (en cuanto a, respecto a).

El elemento causante de la atenuación en el corpus es la presencia, más o menos explícita, de un contenido proposicional negativo o potencialmente negativo que debe ser gestionado de alguna manera por la entidad enunciadora. En unos casos, se controla para evitar efectos no deseados en la recepción de la noticia, como el daño a la imagen de otras personas o de determinados procesos; en otros, para conservar una imagen sustentada 
en la neutralidad y la objetividad. La cautela característica de la atenuación temperativa (Caffi 2007: 259) está orientada aquí, por lo tanto, en dos direcciones diferentes.

En lo que respecta a la dimensión funcional de la atenuación, detectamos en el corpus la presencia de tres funciones: 1) autoprotección, vinculada a la elusión de responsabilidades sobre lo dicho y la corrección política; 2) prevención, principalmente de cara a una eventual amenaza a la imagen de terceras personas y a la posibilidad de rechazo o desacuerdo; y 3) protección sin imagen, relacionada con el interés por controlar el alcance de determinada información. En cuanto a esta última categoría, defendemos su pertinencia por tratarse de casos orientados a controlar el alcance potencialmente negativo del mensaje sin que exista un riesgo para la imagen de las entidades implicadas en la situación comunicativa.

La aplicación de los modelos propuestos nos ha permitido sistematizar las estrategias atenuantes registradas en el corpus y poner de manifiesto algunos aspectos particulares. A su vez, se abren interesantes cuestiones en lo que respecta a la combinación de recursos atenuantes en un mismo enunciado y la posibilidad de determinar jerarquías. Por otra parte, resultaría interesante profundizar en las motivaciones subyacentes en casos de atenuación en los que no existe una amenaza directa a la imagen propia o ajena y comprobar hasta qué punto estos procesos están relacionados con la presencia de una dimensión persuasiva (convencer de la idoneidad o inconveniencia de un estado de cosas) en los textos informativos de prensa.

\section{BIBLIOGRAFÍA}

Albelda, Marta y otros (2014): «Ficha metodológica para el análisis pragmático de la atenuación en corpus discursivos del español (es.por.atenuación)», Oralia, 17, 7-62.

Albelda, Marta y Antonio Briz (2013): «Una propuesta teórica y metodológica para el análisis de la atenuación lingüística en español y portugués. La base de un proyecto en común (es.por.atenuación)», Onomázein, 28, 288-319.

Blum-Kulka, Shoshana (1989): «Playing it safe: The role of conventionality in indirectness», en Blum-Kulka, Shoshana y otros, eds., Cross-Cultural Pragmatics: Requests and Apologies, Norwood, Ablex, 37-70.

Blum-Kulka, Shoshana y otros, eds. (1989): Cross-Cultural Pragmatics: Requests and Apologies, Norwood, Ablex.

Bouzas, Paula (2016): «Análisis del discurso sobre las lenguas del estado español en la prensa hegemónica durante la era Aznar», en Bürki, Yvette y Henriette Partzsch, eds., Redes de comunicación. Estudios sobre la prensa en el mundo hispanohablante, Berlin, Frank y Timme, 157-174.

Bouzas, Paula (en prensa): "Contribución al estudio de los recursos de intensificación en los textos informativos de prensa", in Albelda, Marta y Wiltrud Mihatsch, eds., Fenómenos semántico-pragmáticos de intensificación y atenuación en español, Frankfurt/Madrid, Vervuert/lberoamericana.

Briz, Antonio (2006): «Para un análisis semántico, pragmático y sociopragmático de la cortesía atenuadora en España y América», Lingüística Española Actual, 29/1, 5-44.

Brown, Penelope y Stephen C. Levinson (1978): «Universals in language usage: Politeness phenomena», en Goody, Esther N., ed., Questions and politeness: strategies in social interaction, Cambridge, Cambridge University Press, 56-311.

Caffi, Claudia (2001): La mitigazione. Un approccio pragmatic alla comunicazione nei constesti terapeutici, Münster, LIT Verlag.

Caffi, Claudia (2007): Mitigation, Amsterdam/Tokio, Elservier.

Caffi, Claudia (2013): «Mitigation», en Sbisà, Marina y Ken Turner, eds., Handbook of pragmatics. Pragmatics of Speech Actions, 2, Berlin/New York, De Gruyter, 257-285.

Cornillie, Bert (2016): «Los auxiliares evidenciales en español», en González Ruiz, Ramón y otros, eds., La evidencialidad en español: teoría y descripción. Frankfurt/Madrid, Vervuert/lberoamericana, 227-250.

Ducrot, Oswald (1986): «Esbozo de una teoría polifónica de la enunciación», en Ducrot, Oswald, El decir y lo dicho. Polifonía de la enunciación, Barcelona, Paidós, 175-238. 
Faerch, Claus y Gabriele Kasper (1989): «Internal and external modification in interlanguage request realization», en Blum-Kulka, Shoshana y otros, eds., Cross-Cultural Pragmatics: Requests and Apologies, Norwood, Ablex, 221-247.

Ferrari, Angela y otros (2008): L'interfaccia lingua-testo. Nature e funzioni dell'articolazione informativa dell'enunciato, Alessandria, Edizioni dell'Orso.

Fraser, Bruce (1975): «Hedged performatives», en Cole, Peter y Jerry L. Morgan, eds., Syntax and Semantics, vol. 3: Speech acts, New York, Academic Press, 187-210.

Fuentes Rodríguez, Catalina: «Enunciación, aserción y modalidad, tres clásicos», Anuario de Estudios Filológicos, vol. 27, 121-145.

Grande Alija, Francisco Javier (2002): Aproximación a las modalidades enunciativas, León, Universidad de León.

Lakoff, George (1972): «Hedges: A study in meaning criteria and the logic of fuzzy concepts», en Peranteau, Paul M. et al., eds., Papers for the Eighth Regional Meeting of the Chicago Linguistic Society, Chicago, Chicago Linguistic Society, 183-228.

Prince, Ellen F., Joel Frades y Charles Bosk (1982): «On hedging in physician-physician discourse», in R. J. Di Pietro, ed., Linguistics and the professions. Proceedings of Second Annual Delaware Symposium on Language Studies, Noorwood, Ablex, 83-97. 